\title{
Urban Abacus of Building Energy Performances: a methodological approach
}

\author{
Stefano Pili ${ }^{1,}$, Valeria Fois $^{2}$, EusebioLoria ${ }^{1}$, Caterina Frau $^{1}$ and Pier Francesco Orrù ${ }^{2}$ \\ ${ }^{1}$ Sotacarbo SPA, Grande Miniera di Serbariu, 09013 Carbonia (CI), Italy \\ ${ }^{2}$ DIMCM - Dept. of Mechanical, Chemical and Materials Engineering, University of Cagliari, Italy
}

\begin{abstract}
This paper presents a methodological approach to develop the tool "Urban Abacus of Building Energy performances (Abaco URbano Energetico degli Edifci - AUREE)" for supporting the renovation and energy efficiency enhancement of urban building heritage. The AUREE tool will be based on a Web - GIS GeoBlog portal with some customized interfaces aimed to share the knowledge on urban building heritage and promote the participation of the stakeholders of the urban community. The tool improves citizen and stakeholders awareness on building energy efficiency issues and facilitates the linkage between demand and offer for building companies services via the integration of two main elements: the first is a knowledge layer that represents the actual state of the energy performance of urban building heritage and the second is a spatial based Abacus of the more common building characteristics (structures, plants, uses, ..) and energy retrofitting and renovation technologies. After a touch on the theoretical context, this paper presents a summary of the AUREE general framework and focuses on the methodological approach of the Urban Abacus. The data structure integrates an Urban Building Energy Model (UBEM) in order to support on the energy retrofitting scenario analysis at the level of single building and also for a group of them (city block, district, city). At the end of the paper the furthers research steps have been pointed
\end{abstract}

\section{Introduction: theoretical context}

Energy efficiency enhancement of urban areas is a major key point of the EU 2020 objectives in the new climate package for 2030 because the European building stock is responsible of about $40 \%$ of emissions. Despite of the development of increasingly performing technologies and materials, ancient buildings with limited energy performance, characterized by a considerable average value of energy needs of about $200 \mathrm{kWh} / \mathrm{m} 2 \mathrm{per}$ year [1], represent almost all of the European built heritage. Urban contexts shows complex relationships between material and immaterial components, stratified over time and with limited degrees of freedom. This strongly affects the potential of renovations and energy efficiency improvement of the building heritage and real estate assets so as the renovation of the city infrastructure networks. Therefore, public administrations rarely develop

"Corresponding author: stefano.pili@,sotacarbo.it 
strategic approaches and implement effective methods, leaving the burden of the renovation of a huge and old building stock to the homeowners, supported by more or less effective national incentives that not consider the local building heritage peculiarity. However, an effective urban strategy is essential to cut the consumption of such vast, old and inefficient building heritage and to combine the private investments and public financial resources on urban transformation design programs [2].

The lack of knowledge prevents the development of energy efficiency market for the real estate assets [3] and affects all the stakeholders involved in the building efficiency improvement: the construction sector workers, the building end-users, the investors and the Local Administration (LA). The workers, both the designers and the constructor companies, may not be aware of the available technologies and/or of the knowledge to ensure the correct implementation and commissioning, because on one hand the technologies are rapidly evolving, on the other, they differ in cost and availability in local markets. The building end-users are generally not technical skilled, but they use the indoor spaces ; moreover, in the case of residential assets, they often mach to the key decision makers who invest in energy retrofitting. The investors require reliable perspectives on the technologies effectiveness, which at times needs excessive study and research activities compared to the value of the good and/or to the cost of the technology improvement investment. The decision makers of the Public Administration (PA), as well as the knowledge about new technologies, need to have a sufficiently representative point of view on the building heritage characteristics as a whole and on the related energy consumption, to design effective incentive policies and update the regulatory framework [4].

In order to overcome the barriers towards energy transition of the building heritage, in a holistic perspective of sustainable recovery, the development and testing of methodologies and support tools are becoming increasingly important. They allow to link the perspective of the single building with the strategies at urban or neighbourhood scale, in order to combine the national incentive policies with the bottom-up applications from the building owners and local businesses[5] [6].

The building heritage study about identification of recurring or "typological" characteristics is a practice widely used and addressed in the scientific field [7] [8]. Narrowing down to a group of buildings sufficiently homogeneous by type (schools, historic buildings, offices, etc.) or by territorial context (city, region, climate, etc.), it is possible to define guidelines based on the specificity of the heritage and on the local availability of consolidated technologies. [9].

The progressive digitalization of the construction process, through the diffusion of Building Information Modelling (BIM), and the opportunities offered by latest generation building smart sensors (IOT, smart meter, etc.), offer new tools to promote quality of the management. This became true mainly in the context of efficiency improvement and renovation of buildings characterized by greater complexity and value (public buildings, shopping malls, large housing complexes, etc.)[10].

Thanks the ever-increasing diffusion of geographic open data and the development of effective tools, examples of methodologies aimed at support of urban energy planning through Urban Building Energy Modelling (UBEM) approaches, are increasingly numerous [11]. In general, these tools incorporate an Urban Energy Model (more or less complex) to estimate buildings energy needs and procedures to estimate the potential (theoretical, technical, and exploitable) of various efficiency technologies and/or design scenarios. They differ significantly from each other in terms of availability of the basic data, reliability and detail (temporal, spatial, etc.) of the results, complexity of the algorithms and type of approaches to modelling (top down, bottom up, etc.) [12]. One of the main differences is the type of approach to energy calculation [13]: statistical, engineering-analytical or mixed. 
Statistical models use different predictive inferential techniques to derive energy demand from a group of known variables (type of buildings, user profile, series of consumption of a sample of units, etc.). These models can very well represent the trend of real demand as they implicitly incorporate the "human" variable [14], but show some limits in the evaluation of technological innovations effects as they are not representative of physical phenomena. Furthermore, the consumption data required for modelling are not always available. On the contrary, analytical models simulate theoretical energy needs starting from the physical characteristics of the heritage (geometries, materials, plants, etc.), using simulative models [15]. These models are ideal for evaluating ex ante design scenarios, but their reliability demand prediction significantly depends on accuracy of the baseline data and on the algorithms adopted. Many literature examples in order to exploit the strengths of both calculation methods, adopt mixed approaches that involve the use of simulation for a real buildings sample or for building types at the beginning and then the use of statistics techniques to up-scale the results to all the building stock [16].

UBEM are tools mainly oriented to urban or suburban scale that incorporate the geographical dimension of information useful for relating to the other traditional urban planning typical themes and to spatial represent the energy demand. GIS tools are the ideal environment for managing and representing geographical information. Many methodologies use them to obtain and pre-process the base data and represent the results; using external resources for energy modelling usually based on dynamic calculation engines [17]. On the other hand, other methodologies fully integrate the procedures with simplified models [18].

In general, complex models, according to a better reliability of the results, require more data and onerous activities of results analysis. Less complex models adopt several simplifying hypotheses, therefore they require fewer resources, and have usually more repeatable procedures. However, the uncertainty of the initial assumptions in this case burdens the results [19]. One of the most used simplifying approaches is the "archetype", which involves the study of a representative sample of the heritage, in order to explicate some elements that characterize the energy need, and the use of appropriate methods (analytical, statistical, etc.) to extending the results of the sample to all similar elements present in the city.

The Tabula EU Project [20] adopts an archetypal approach based on energy standard calculation in order to estimate the energy retrofit potential of the European building heritage. It defines some typologies for each national climate zones based on year of built and building typology (block, row, detached ,..). The retrofit potential has been studied for each typology and then scaled to national level by census stats on building heritage.

Some recent UBEM approach addressed to urban scale [14] [16] use a mix of simulation and statistical techniques and adopt the TABULA data base and census stats as input knowledge for the building physical data (envelope, plant, ..) and to define retrofit hypotheses. The accuracy of the energy demand estimation is ensured by appropriate calibration technique based on consumption data, but input knowledge on building heritage are highly typological. The focus seem to be more on the accuracy of the demand estimation than the definition of the building heritage characteristics that could direct affect the renovation and energy retrofit potential. This mix of physical characteristics and uses of the building is important to define reliable retrofitting hypothesis linked to local market and heritage specificity and is a relevant background knowledge for urban detailed planning activities. Moreover in the majority of the Italian urban contexts, mostly in the average small sized cities, energy consumption data are not available and generally unlikely could have enough resources to manage complex software that involve highly specialized expertise. Therefore the definition and testing of methodologies that could be integrated on the more common urban planning practice is still an open field of research 


\section{Methodology: general framework}

There are many literature exempla of guidelines or quality protocols on the topic of energy efficiency specified to particular types of buildings or focused on a more or less wide territorial area. However, these are generally not integrated into a geographical based tool that supports in the knowledge sharing and favours in the updating procedures. The objective of this work is to define and test a methodology that use the similarities between the UBEM archetypal approach for the study of energy aspects and the typological one adopted to define design guidelines, in order to create a sort of geographical abacus of the building heritage of an urban centre oriented towards buildings renovations and energy retrofitting. The UBEM archetypal approach here proposed is based on local knowledge on building heritage that could be defined from urban planning documentation, maps and specific open data availability. The idea is to develop an approach that adopt mainly tools and procedure already known on the fied of urban planning practices in order to facilitate the integration of energy issues in the management of the urban transformation that generally refers to the single building scale. Energy Retrofitting hypotheses have to deals to local regulation framework that is addressed to the characteristic of the single building (historic value, envelope shape and specific elements, real estate property, ...). This kind of knowledge approach is also the baseline data for the definition of strategies and projects for urban block and/or district renovation that traditionally involve others multidisciplinary themes.

The methodology is designed to be implemented also in medium and small sized Italian urban contexts, which are often characterized by limited economic and human resources. Therefore the main basic data of the methodology are the territorial OPEN data generally available in the national territory (regional GeoDB, ISTAT data, documentary knowledge related to urban planning, etc.), some knowledge on energy performances of construction materials and plants and easy survey activities. Moreover, in order to favour the replication of the methodology and the exportability of the models, the methodology will be implemented on GIS environment mainly adopting tools and procedure which are already partially disseminated among practitioners and PA.

The research therefore focuses on the development of a methodological approach that integrates the knowledge approach of a typological study with a UBEM and the capability to represent and sharing of a geographical information system by defining an "Urban Energy Abacus of Buildings - AUREE". Therefore, the methodology is mainly articulated on some main topics:

- definition of a survey methodology and representation approach of the building heritage;

- development of the approach to City Modeling, Energy modelling e spatial data modelling;

- definition and testing of the communication tool, the portal and the GUI.

The tool could be basically defined as a protocol of analysis and representation of the building heritage, based on locally available open geographical data (SDI), easy urban survey activities and sensors; combined in a communication and involvement tool developed on WEB GIS infrastructure addressed to local actors. It is a SMART Planning approach, which integrates the open data available with the emerging data harvesting techniques linked to SMART Buildings, in order to constitute a common platform for the creation, synthesis and sharing of knowledge to support the processes of renovation and energy efficiency improvement of public and private building stock. 
The City Modelling is mainly aimed at the definition of four geographical groups of layers, which constitute the baseline data for the services of the the portal and for the interaction with local actors (Fig. 1):

- context layers: study and representation of the elements of the urban context that can affect the sustainable recovery of the heritage;

- public buildings layer: support tools for quality energy management of the building $t$ and for designers through guidelines and tools (BIM);

- layer of the built heritage: representation of the building heritage with GIS approaches about energy efficiency and sustainable recovery potential through an UBEM;

- geographic abacus: collects and rearranges knowledge on recurring elements of the building heritage on a GIS spatial environment, promoting its diffusion also to a non-technical public.

Considering the differences in value and complexity between public buildings and the rest of the heritage mainly dedicated to private residence, the tool adopts two different knowledge and representation approaches. In the case of public buildings, a protocol based on energy audit procedures and sensors monitoring is proposed. This approach aims to geographic representation and sharing of energy data and building values, and at supporting the phases of project, construction, monitoring and management through BIM approaches.

For residential built heritage, an approach based on the study of building typologies and its recurring elements is proposed, coupled with the development of a UBEM, useful for assessing the energy retrofit potential of the building heritage.

The abacus is a spatial database that contains the typological information of the recurring elements of the building heritage (structures, systems, usage profiles, and retrofit interventions) linked to the geographical reference element (the building). It aims to support the project activities and the evaluation of various alternative of building renovations through the geographical representation of the most widespread architectures and the proposal of adequate technologies related to the local market.

The context layers are an open set of themes, also not directly related to the aspects of energy efficiency, which can however influence the renovation potential. It is not a simple collection of regulatory constraints, but it creates a background knowledge shared between decision makers and operators in the building sector to direct towards the development of holistic approaches to the project on an urban scale.

The representation and sharing tool is based on a multi-user web infrastructure (dedicated portal), capable of involving the different types of interested stakeholders. It is configured as an interface that actors can use to explore and consult the knowledge base, but also to provide their feedback and contribution in compliance with their respective interests and access levels. As a preliminary, it may include: documentary and informative material, the case studies developed, the data of the recurring typological building elements, lists of energy efficiency technologies and virtuous practices carried out, summary statistics and indices on the state of the energy performance of the real estate assets, statistics and data on the monitoring of energy consumption and interventions carried out on public buildings. In this way, a dynamic shared knowledge will be created, which feeds and updates its contents based on the interaction of the users of the portal and with the data obtained from the sensors.

The research purpose is therefore to define in detail the procedures and tools that connect the different thematic contents (public buildings layer, building heritage layer, geographical abacus, context layer, data collected by the sensors) to the representation and interaction with local actors through the portal (fig. 1). 
This article will expose in more detail the methodological approach developed for the the Abacus of the residential building heritage with its relations with the UBEM and with the participatory interfaces, leaving the description of the other elements of the AUREE project to furthers publications.

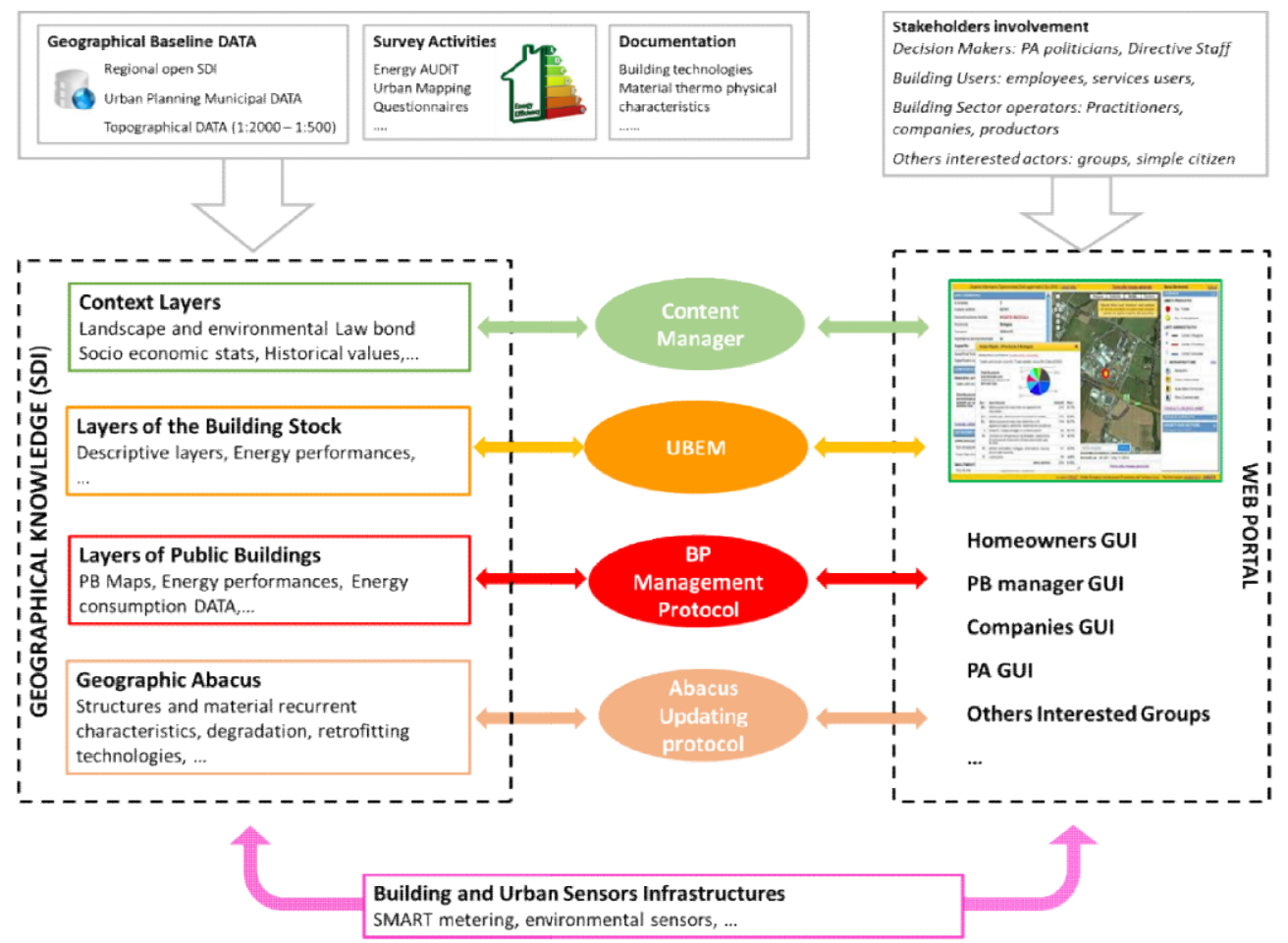

Fig. 1.Framework of the AUREE project

\section{UBEM methodological approach}

The residential building stock layer is the main mean of interaction with citizenship, as all homeowners (or occupants) of the building real estate can theoretically be interested. The Local Administration can also use this knowledge content to support the drafting of urban renovation programs and project geared towards energy efficiency. The information concerns the main features of the current state of the building heritage about energy performance aspects, anyway other issues can be integrated (Indoor environmental quality, Comfort, Materials Life Cycle, etc.). This spatial layer is linked to the development of an Urban Building Energy Model (UBEM) approach aimed to supports the definition of the performance aspects of the heritage, providing a calculation engine to simulate scenarios at the scale of the individual building and / or for groups of them.

The proposed approach is based on a bottom-up engineering model, set in a GIS environment, for the definition of the energy performance of the building heritage capable to make calculation for each building and simulating the effect of retrofitting technologies. The UBEM is designed to create the base information for a communication and dissemination of knowledge instrument, so it was decided to refer to the regulatory framework [20] introduced by the Italian Energy Performance Certificate (EPC), as it is now accepted by technicians and designers and increasingly popular among decisionmakers and ordinary citizens. 
The model input data are: a topographic DB,with a precision generally available in the national territory as it is used for urban planning activities (scale 1: 2000), a typological study of the local building heritage and some expeditious urban survey activities. Although the use of consumption monitoring systems (SMART meters) is becoming more widespread, the cases with sufficiently homogeneous and complete DB are still limited to some best practices related to energy services municipal networks or to specific research projects. Therefore, the proposed approach does not use consumption data as starting information, but calculates an energy performance with the available basic data, trying to involve users for more accurate information recovery in order to refine the model iteratively. Leaving to an another publication the detail of the algorithms (fig. 2), the GIS tool first obtains the geometric data of each building (dispersing surfaces, orientation and shading of the facades, heated volumes and useful surfaces, ..) via some geo processing procedure, then it calculates the energy needs of the envelope and the primary energy need by applying an adaptation of the technical standard (UNI 11300 parts 1-2-3-4). The results of the calculation are used to create maps and content for the portal aimed at involving the homeowners of the real estate units. These, through specific interfaces, will be able to specify some information (e.g. type of systems, usage profiles, consumption) and obtain personalized retrofit suggestions based also on the new data entered. In this way, the typological information defined initially can be gradually improved through the voluntary participation of the users of the portal, also increasing the reliability of the model and the significance of the current state by acquiring data on consumption and on user preferences. The local typological study is therefore the basis for the definition of the input data of the UBEM energy calculation referring to the building - plant system (structures, materials, plants, etc.), but it also constitutes the basis to inform towards the recovery interventions and energy efficiency proposed in the Abacus.
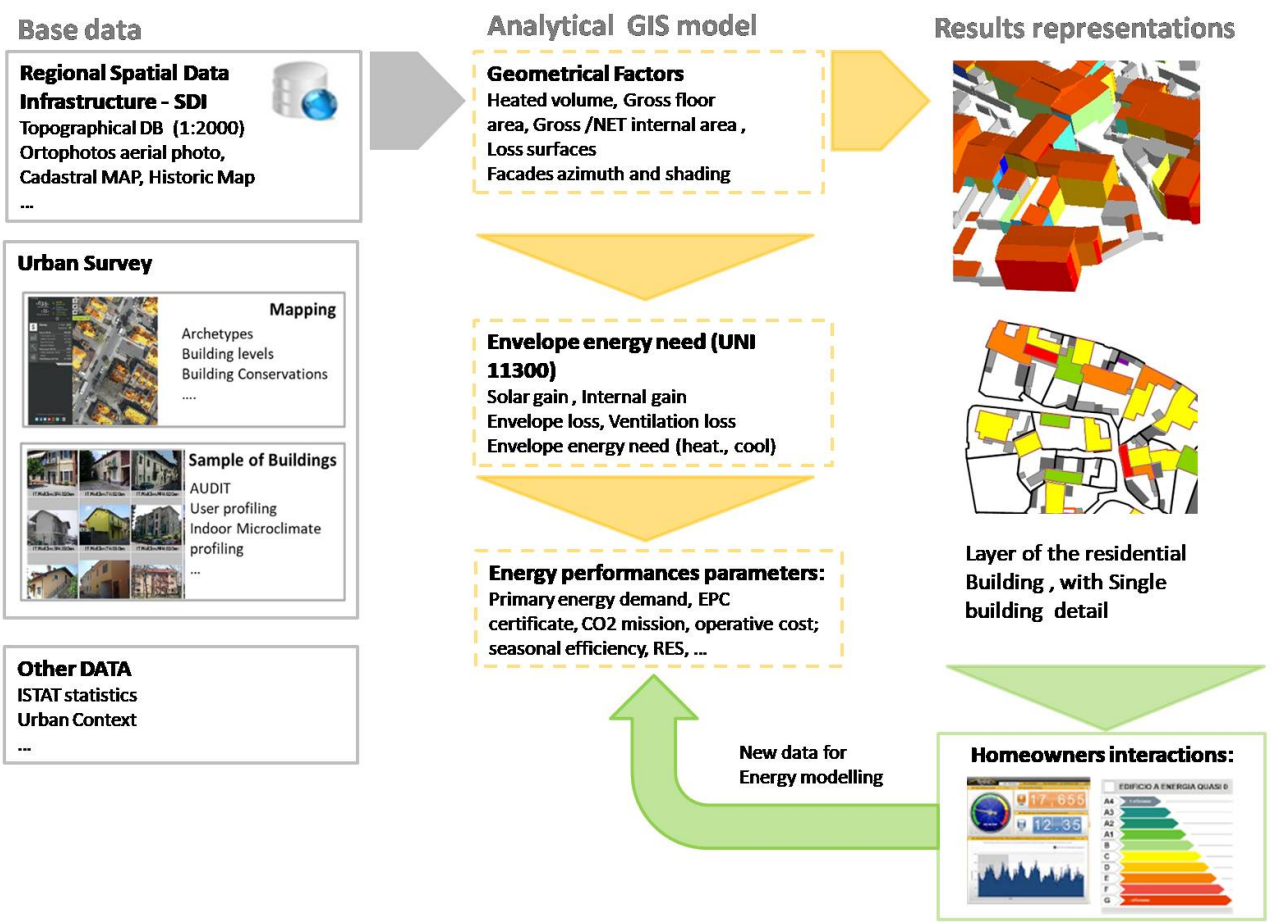

Fig. 2. The framework of the Urban Building Energy Model 


\section{The Building Urban Abacus approach}

The proposed study is focused on the development of a dynamic Urban Abacus aimed at sharing data and information on local building heritage to involve a full group of stakeholder. The basic idea is to develop a framework for investigation and testing of environmetally-friendly design, technologies and practices solutions to be performed in archetypes. Thus, the Abacus represents a valuable communication tool between professional and homeowner (i.e, the stakeholders) contributing to new knowledge and understanding (fig. 4). To this end, it has been tailored and customized as a function of different constructive elements and architectural types such as: building configuration, typologies and retrofit, renovation technologies, user's thermal comfort, heating and cooling systems. The main goal is to provide a better insight into the factors that affect the building heritage sustainability in terms of energy efficiency, environment, economics and social well-being. The dataset achieved by the proposed methodology allow to define strategies and solutions for building possessing similar characteristic by the integration into the bottom-up approach UBEM.. Moreover, the Abacus offers the opportunity to store these dataset aiming at providing a feasible tool for economic incentives of Agenda 2030 which encourage existing building to go green and be more energy efficient.

\subsection{Stakeholders}

The stakeholders are identified as the PA, the economic actors and building owners. The Public Administration will be able to use the Abacus data and information to develop a useful background information on both building and construction typologies and their energetic characteristics aimed at providing proper and efficient solutions. To this end, in order to promote green construction, governments provide grants or tax incentives affecting both the individual building level and urban realms.

The economic operators involved in the retrofit process are identified in designers and companies. The Abacus can be a valuable tool to investigate the performance of analyzed buildings including built quality, energy consumption, user satisfaction, comfort and health. Moreover, as experts in the field, they could be propose solutions based on market and technological trends.

Finally, the private owners can use Abacus to develop an informed decision-making to find optimal solution based on the knowledge of energy-related characteristics their own building.

\subsection{Abacus structure}

The urban abacus consists of three levels of implementation characterized by data updating, changes or integration (Fig. 3).

First level is a database that draw the recurring "typological" characteristics of the building heritage related to the geographical reference points

- archetypes

- building types

- year of built

- geographical location

- surface

Second level are the users interfaces based on a feedback GUI to collect the data set by the homeowners: 
- building structures: e.g. changes building envelope - windows etc.

- plant types

- renewable energy - F.E.R.

- utility and maintenance cost

- user profiling

- primary energy demand

- real and expected microclimate comfort

Third level is the project level. Two different retrofit strategies/criteria are defined by considering the building typologies collected and the solutions described in the previous levels:

- light innovation

- deep innovation

Archetypes

Buiding details

Year of built

Geographical location

Surface

Building structures

Plan types

F.E.R. - renawable

energy

ut ility and maintenance

cost

User profiling

Primary energy demand

Real and exepected

microclimate comfort

light innovation

deep innovation

\section{Sotacarbo SPA}

DIMCM

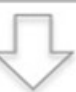

Similar best practice
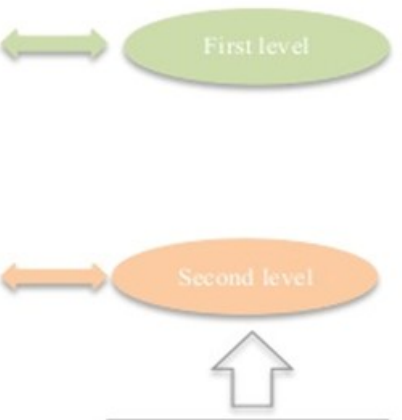

New data

Homeowners

Pubblic

Administration

Economic operators

Third level

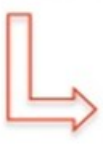

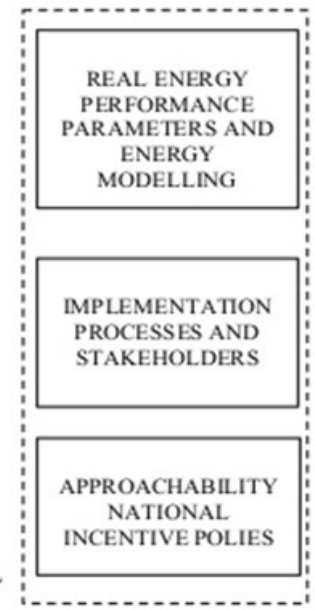

Fig. 3 Levels of Urban Abacus approach

The retrofit criteria reported in the Abacus are a composition of different technologies and consider the different economic incentive tools and the specific requirements to benefit from them. The light renovation involves the implementation of actions used strategically to lower energy consumption with a minimum impact on the structure by a small investment, such as the thermal energy system replacement and the adoption of thermostatic valves. On the other hand, deep renovation involves a substantial retrofit of the 
building by means of envelope performance improvement, system upgrading, volumetric addictions among others. Furthermore, the Abacus not only takes into account the environmental and energetic aspect but also the economic feasibility of the possible interventions. Therefore, it represents a proper methodology to drive form a strategic point of view the implementation of energy efficiency actions for building heritage to meet the energy targets of 2030 Agenda.

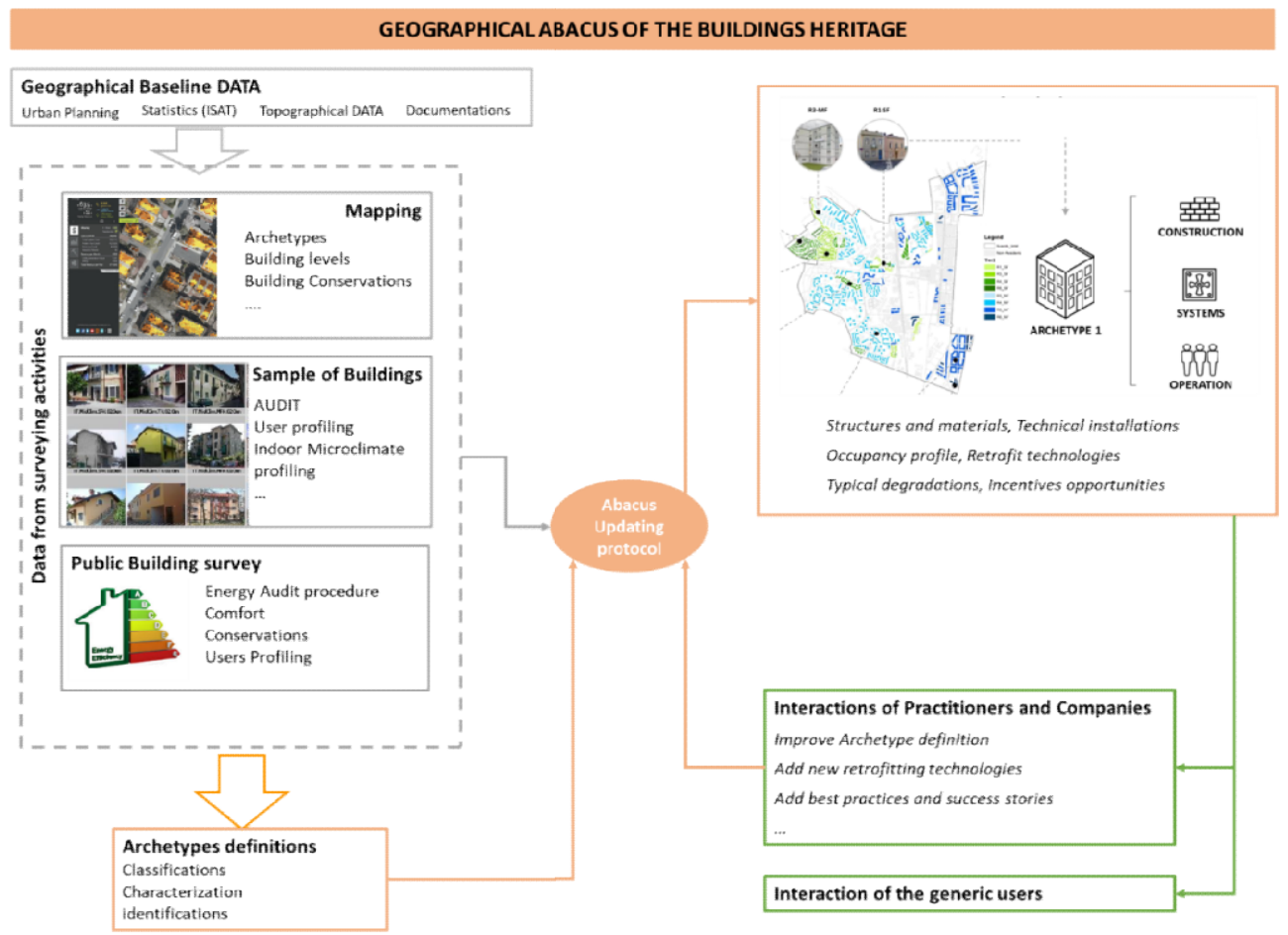

Fig. 4 Schematic of a typical sheet Urban Abacus approach

\section{Conclusions}

The paper shows the overall structure of a tool aimed at supporting the buildings energy efficiency process. The tool refers, not only to the scale of generic or detailed urban planning, but also to medium-small size urban contexts, characterized by limited economic resources and a lack of basic data.

One of the innovative aspects is the application of a common preliminary knowledge base for the UBEM archetypal approach and to define retrofitting guidelines strongly linked to the local context and equipped with spatial reference in order to simplify data searching and finding. The energy model integrated into the UBEM makes more interactive the guidelines suggestion to track most effective technological solutions defined by the specific characteristics of the building. On one side, guidelines assumption are $a$ priori based on the typological study of the building heritage, on the other they are partly obtained by the energy model based also on the feedback from the private property owners.

The tool, aimed at different stakeholders, each with their own role, contributes to develop a unique knowledge fact-finding database, from the awareness of the current state to the sharing of possible alternative designs. The interaction between the property owners and the 
workers in the sector (companies, professionals, etc.) could lead to many advantages. On one hand it allows to tune the model with more accurate information on the buildings and on the use profiles of the heritage (energy consumption, comfort, etc.), on the other to keep the abacus updated through the availability of the technologies and examples of local best practices.

The geographical scale allows the model to identify macro areas with similar characteristics and to define synthetic indices to compare also different contexts. These information layers are the key elements to link the single action project addressed to the building to a urban scale strategic dimension.

The research is ongoing and it is fine-tuning a case study to test the advantages and disadvantages of the methodology and to define the procedures. Carbonia, located in the south western of Sardinia with about 28,000 inhabitants, is the selected city. It was founded in the 1930s to support the mining activities of the Grande Miniera di Serbariu. It represents an ideal case for testing the methodology, because of a rather uniform, consistent and welldocumented real estate heritage, both in the historical part of the city (fascist's foundation buildings) and in the more recent areas mainly implemented through planned expansions, that are still being completed.

The research framework and the article structure is to be attributed to Pili S., Frau C. and Orrù P.F. are the research groups supervisors. Pili S., Loria E. and Frau C., wrote the texts of paragraphs 1. 2. 3. and Fois V. and Orrù PF the paragraph 4, while the conclusions are of the whole group. The resources for this publication has been given by the Sotacarbo SPA, within the "Research of Electric System" project funded by "Ministry of Economic Development" CUP: I34I19005780001

\section{References}

1. https://www.odyssee-mure.eu/publications/br/energy-efficiency-trends-policiesbuildings.pdf

2. P. De Pascali, M. Reginaldi, V. Alberti, D. De Ioris, Applicazioni di sostenibilità ecoenergetica per la riqualificazione urbana, Orienta Edizioni (2014), ISBN-13: 9788896467312

3. L. Kranzl and the ENTRANZE consortium, Laying Down The Pathways To Nearly Zero-Energy Buildings A toolkit for policy makers, Final Report of the Policies to enforce the transition to nearly zero energy buildings in the EU-27 (ENTRANZE) founded by IEE (2014), http://www.entranze.eu/pub/pub-policies

4. P. Caputo, G. Pasetti. Overcoming the inertia of building energy retrofit at municipal level: The Italian Challenge. Sustainable Cities and Society, 15 (2015), pp. 120-134. https://doi.org/10.1016/j.scs.2015.01.001

5. C. Del Mastro, G. Mutani, S. P. Corgnati, A supporting method for selecting costoptimal energy retrofit policies for residential buildings at the urban scale, Energy Policy 99 (2016) , 42-56, DOI: 10.1016/j.enpol.2016.09.051

6. P. Caputo, G. Costa, S. Ferrari, A supporting method for defining energy strategies in the building sector at urban scale, Energy Policy, 55 (2013), 261-270, DOI: $10.1016 /$ j.enpol.2012.12.006

7. L. De Santoli, Guidelines on energy efficiency of cultural heritage, Energy and Buildings 86 (2015) 534-540, http://dx.doi:10.1016/j.enbuild.2014.10.050 
8. F. Ascione, N. Bianco, R.F. De Masi, F. De Rossi, G. P. Vanoli, Energy retrofit of an educational building in the ancient centre of Benevento.Feasibility study of energy savings and respect of the historical value, Energy and Buildings Volume 95 (2015), 172-183, http://dx.doi.org/10.1016/j.enbuild.2014.10.072

9. K. Kass, A. Blumberga, D. Blumberga, G. Zogla, A. Kamenders, E. Kamendere, Preassessment method for historic building stock renovation evaluation, International Scientific Conference "Environmental and Climate Technologies", CONECT 2016, 12-14 October 2016, Riga, Latvia, Energy Procedia 113 (2017), 346 - 353, https://doi:10.1016/j.egypro.2017.04.004

10. A. L. Ciribini, BIM e digitalizzazione dell'ambiente costruito, Grafill Palermo (2016),

11. C. F. Reinhart, C. Davila, Urban building energy modeling - A review of a nascent field, Building and Environment 97 (2016), 196-202. https://doi.org/10.1016/j.buildenv.2015.12.001

12. Lukas G. Swan, V. IsmetUrgursal, Modeling of end use Energy consumption in the residential sector: A review of modelling techniques, Renewable \& Sustainable Energy Reviews 13 (2009) 1819-1835.DOI:10.1016/j.rser.2008.09.033

13. N. Abbasabadi, M. Ashayeri, Urban energy use modelling methods and tools: A review and an outlook, Building and Environment 161 (2019), 106 - 270, https://doi.org/10.1016/j.buildenv.2019.106270

14. S. Torabi Moghadam, J. Toniolo, G. Mutani, P. Lombardi, A GIS-statistical approach for assessing built environment energy use at urban scale, Sustainable Cities and Society 37 (2018), 70-84, https://doi.org/10.1016/j.scs.2017.10.002

15. D. Robinson, F. Haldi, J. Kämpf, P. Leroux, D. Perez, A. Rasheed, U. Wilke, CitySim: comprehensive micro-simulation of resource flows for sustainable urban planning,Building Simulation Eleventh International IBPSA Conference, Glasgow, Scotland July 27-30, (2009). https://infoscience.epfl.ch/record/148717

16. R. Nouvel, A. Mastrucci, U. Leopold, O. Baume, V. Coors, U.Eicker, Combining GISbased statistical and engineering urban heat consumption models: Towards a new framework for multi-scale policy support, Energy and Buildings 107(2015), 204-212. https://doi.org/10.1016/j.enbuild.2015.08.021

17. Y. Chen, T. Hong, M.A Piette, City-scale building retrofit analysis: A case study using CityBES, Build. Simul. (2017), San Francisco, CA, USA. https://citybes.lbl.gov/BS2017_CityBES_Paper_Final.pdf

18. S. Gadsden, M. Rylatt, K. Lomas, Putting solar energy on the urban map: a new GISbased approach for dwellings, Solar Energy 74 (2003) 397-407. DOI: 10.1016/S0038092X(03)00190-7

19. J. Keirstead, M. Jennings, A. Sivakumar, A review of urban energy system models: Approaches, challenges and opportunities, Renewable and Sustainable Energy Reviews 16 (2012) 3847-3866; https://doi:10.1016/j.rser.2012.02.047

20. https://episcope.eu/building-typology/tabula-webtool/

21. D.Lgs. 192/2005 (and s.m.s) and Italian Standard UNI/TS 11300:2014. https://www.cti2000.eu/la-uni-ts-11300/ 